Supplementary Information

\title{
Mesoporous MCM-48 aluminosilica oxynitrides: synthesis and characterization of bi-functional solid acid-base materials
}

\author{
Yongde Xia and Robert Mokaya*
}

School of Chemistry, University of Nottingham, University Park, Nottingham NG7 2RD, $U$. K.

\footnotetext{
* To whom correspondence should be addressed. E-mail: r.mokaya@nottingham.ac.uk.
} 


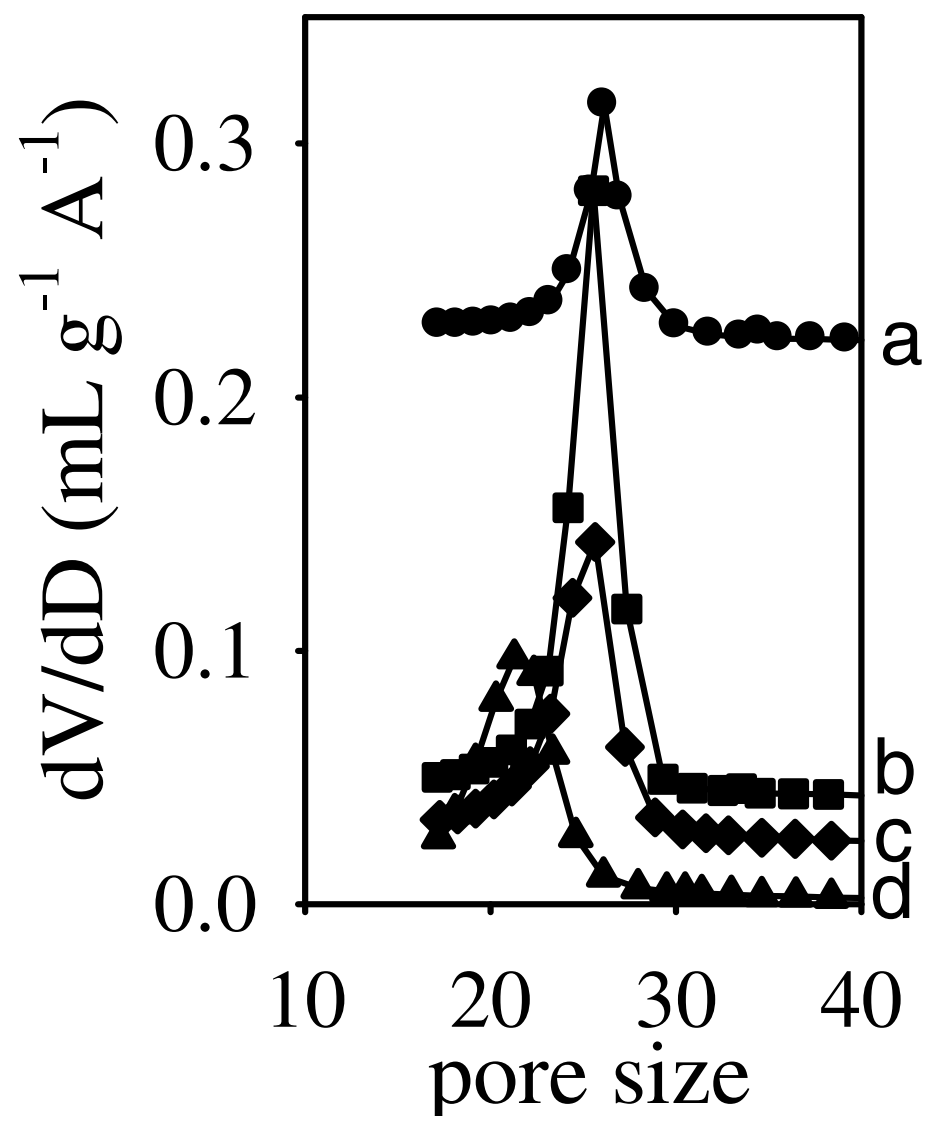

Supplementary Figure 1. Pore size distribution curves for pure silica Si-MCM-48 (a) and directly synthesized Al-MCM-48 samples of various Si/Al ratios: (b) 33.1 (Al-MCM-48A); (c) 20.5 (Al-MCM-48B) and (d) 10.1 (Al-MCM-48C). 

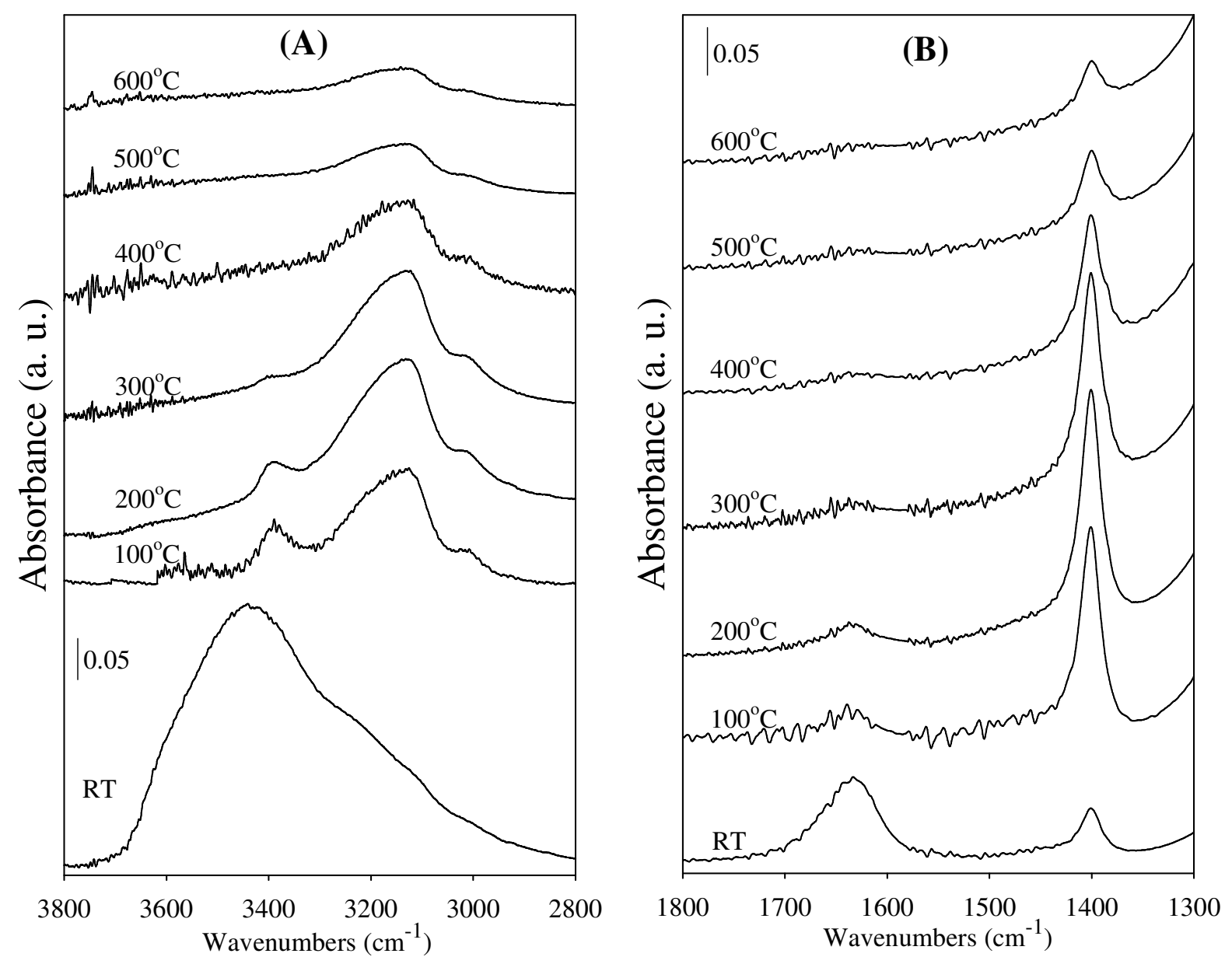

Supplementary Figure 2. Infrared spectra of aluminosilica oxynitride (sample NAl-MCM48A) derived from directly synthesized Al-MCM-48, as a function of evacuation temperature. The spectra are displayed in two regions: (A) $3800-2800 \mathrm{~cm}^{-1}$ and (B) $1800-1300 \mathrm{~cm}^{-1}$. 


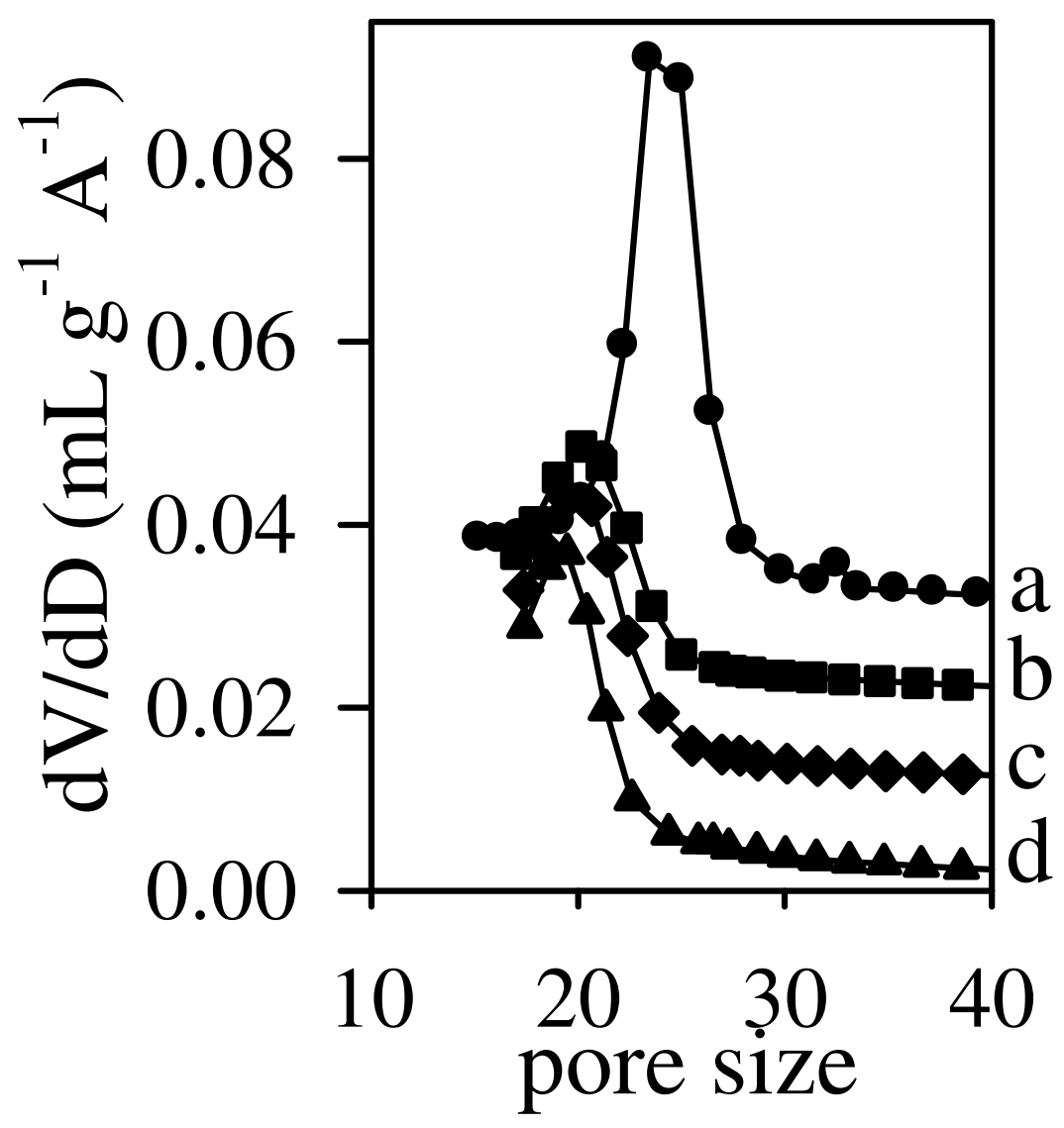

Supplementary Figure 3. Pore size distribution curves for pure silica Si-MCM-48 (a) and Algrafted MCM-48 samples of various Si/Al ratios: (b) 23.8 (GAl-MCM-48A); (c) 16.0 (GAlMCM-48B) and (d) 8.9 (GAl-MCM-48C). 

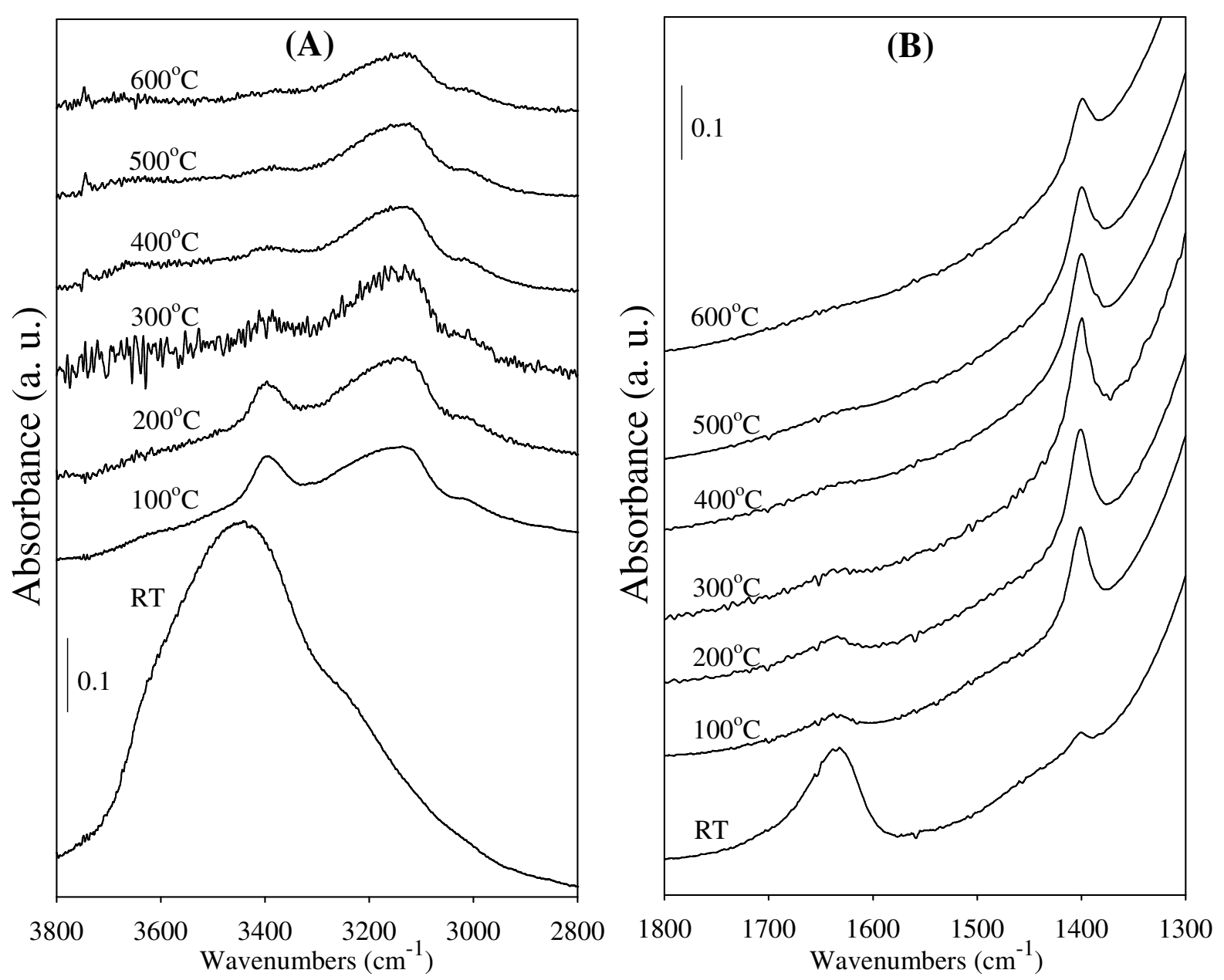

Supplementary Figure 4. Infrared spectra of aluminosilica oxynitride (sample NGAl-MCM48A) derived from Al-grafted MCM-48, as a function of evacuation temperature. The spectra are displayed in two regions: (A) $3800-2800 \mathrm{~cm}^{-1}$ and (B) $1800-1300 \mathrm{~cm}^{-1}$. 